\title{
Simulation and Modeling Time Response of Double Carrier Avalanche Photodiodes
}

\author{
Helmy Fitriawan
}

Faculty of Engineering, University of Lampung, Jl. Sumantri Brojonegoro No. 1, Bandar Lampung, 35145

Email: helmy.fitriawan@eng.unila.ac.id

Article Information
Received:
1 October 2020
Received in revised form:
8 November 2020
Accepted:
5 December 2020
Volume 2 , Issue 2 , December 2020
pp. $102-106$
C Universitas Lampung
http: / dx. doi.org/10.23960/jesr.v2i2.45

\begin{abstract}
Avalanche photodiodes (APDs) is a particularly sensitive semiconductor device that employs the photoelectric effect to convert light into electricity. APDs can be used in some typical applications, i.e. imaging, optical fiber communications, range finding, laser scanners and laser microscopy. In APDs, avalanche multiplication occurred due to impact ionization when the devices operating at high electric fields. Unfortunately, avalanche multiplication decreases the time response of APDs. The time response of an APD can be characterized by its current response which is represented by the mean current as a function of time. This paper discusses a method to estimate the time response of double-carrier multiplication avalanche photodiodes (APDs). The model, called The Random Path Length $(R P L)$, generates random path length for a carrier to impact ionize and takes account of dead space distance into the calculation. Dead space distance is the minimum distance to travel by a carrier to gain the adequate energy to start first ionization. The RPL is applied into an ideal structure which is assumed has a dimensionless multiplication length, $w=1.0$, with electrons and holes moving in constant speeds, $v_{e}=v_{h}=v$, for various dead spaces distances, $d^{*}$. In this research, a computer code is generated to compute the mean impulse response, $i(t)$, and the standard deviation, $\sigma(t)$, of APDs all as a function of time.
\end{abstract}

Keywords: avalanche photodiodes, dead space, random path length, time response

\section{INTRODUCTION}

$\mathrm{T}$ he optical receiver is a device which is responsible to convert information or energy from optical form into electrical signal and recover the data transmitted through optical communication system [1]. One device used as an optical receiver is photodiode, which produce current relative to the light power imposing on the diode. When incident light (photon) illuminate the junction of photodiodes on one side, the photon will be absorbed and an electron-hole pair is created. Electrons and holes created inside the region accelerate in opposite directions, creating flow of current in the external circuit, which is proportional to the incident optical power. Avalanche photodiode (APD) is photodiode with internal gain, which has ability to increase the number of electron-hole pairs per injected photon. Their output current is extremely higher compared to those of other types of photodiodes. The avalanche multiplication is the fundamental mechanism in the operation of APDs.

APDs are preferred to use among the optical receivers in optical communication systems [2-4]. APDs have excellent internal gain, which extensively increases receiver sensitivity and reduces the necessary for optical pre-amplification. The process of carrier impact ionization, which is a phenomenon behind the production of gain in APDs, results in fluctuations in the multiplication gain and also in the response of time [5-6]. This fluctuation introduces noise which can be misunderstood as missing of information in communication systems.

The estimation of multiplication gain, time response and excess noise factor must account a carrier history to obtain more accurate results. The accurately prediction of time response of APDs in this paper also takes account the dead space distance. Dead space is a minimum distance required to travel by a carrier to obtain enough energy to impact ionizes [7]. It has been showed that dead space is essential for estimating the multiplication gain and excess noise factor of APDs [8].

In this paper, the random path length (RPL) model is developed to estimate the number of carriers produced in the gain region. The model takes account the dead space distance into the calculation. The data of carrier number is used to predict the time response of APDs. Results of estimation are presented in graphs of mean 
impulse response, $i(t)$, and its standard deviation, $\sigma(t)$, all as a function of time. The rest of the paper is explained as follows. Next section describes the model used in the simulation as well as the current response calculation. Some simulation results and discussions are presented in section III. Finally, Section IV concludes the study.

\section{MODELS AND METHODS}

\section{A. Random Path Length Model}

The random path length (RPL) model of avalanche process is developed to model the performance of avalanche photodiode [9-10]. The model generates a randomly ionization path length and include the dead space into the calculations of multiplication and noise of avalanche photodiodes. In the RPL model, the electrons and holes traveling a random distance before they impact ionize. These random distance variables are assumed to be statistically independent and have probability density functions. The probability density function for an electron to initiate impact ionization after traveling a distance $x$ in a uniform electric field, E, is given by

$P e(x)=\left\{\begin{array}{cl}0, & x \leq d_{e}{ }^{*} \\ \alpha^{*} \exp \left[-\alpha^{*}\left(x-d_{e}{ }^{*}\right)\right], & x>d_{e}{ }^{*}\end{array}\right.$

Where $d_{e} *$ is the electron hard threshold dead space, and $\alpha^{*}$ is ionization coefficient for electron after dead space occurred. The value of $d_{e}^{*}$ is determined by electron charge, $e$, ionization threshold energy, $\varepsilon_{\mathrm{th}}{ }^{*}$, and electric field, E.

$d_{e}{ }^{*}=\frac{\varepsilon_{t h}{ }^{*}}{e E}$

Hence, the probability for an electron to ionize as a given position does not depend only on applied electric field, as in the local model, but also on its history. From (1), the average distance between the position where the electron impact ionize is given by the following equation,

$<l>=\int_{0}^{\infty} x P_{e}(x) d x=\frac{1}{\alpha^{*}}+d_{e}{ }^{*}$

Since the ionization coefficient, $\alpha$, is the inverse of average length of the electron impact ionize, therefore [11]

$\alpha=\frac{1}{d_{e}^{*}+\frac{1}{\alpha^{*}}}$

The probability that a carrier travels distance $x$ without generating secondary electron-hole pair $\mathrm{S}_{\mathrm{e}}(x)$ can be obtained from (1).

$\operatorname{Se}(x)=\left\{\begin{array}{cl}1 & x \leq d_{e}{ }^{*} \\ \exp \left[-\alpha^{*}\left(x-d_{e}{ }^{*}\right)\right], & x>d_{e}{ }^{*}\end{array}\right.$
In order to get a random path length for electron to impact ionize, $l_{e}$, in this RPL model, a random number, $r$, between 0 and 1 is injected into $\mathrm{S}_{\mathrm{e}}(x)$ in (5).

$r=\exp \left[-\alpha^{*}\left(l_{e}-d_{e}^{*}\right)\right.$

$l_{e}=d_{e}{ }^{*}-\frac{\ln (r)}{\alpha s^{*}}$

Thus by substituting, $\mathrm{P}_{\mathrm{h}}(x), \mathrm{S}_{\mathrm{h}}(x), \beta, \beta^{*}, d_{h} *$ and $l_{h}$ for $\mathrm{P}_{\mathrm{e}}(x), \mathrm{S}_{\mathrm{e}}(x), \alpha, \alpha^{*}, d_{e}{ }^{*}$ and $l_{e}$, the RPL model for hole can be obtained. By using this model, we can estimate multiplication and noise factor in double carrier avalanche photodiodes by involving the dead space model. Under consideration is a double carrier multiplication APD with a multiplication region of $w$ and a uniform electric field. To begin avalanche multiplication process, an electron is injected at position $x=0$ then traveling a random distance to impact ionize, according to (7). Hence, at this position in addition to the original electron, there will be a new electron and a hole is generated. All three carriers then will travel new random length, selected independently, to initiate impact ionization and produce many secondary carriers. All secondary holes and electrons repeat each this process independently until all carriers have left the gain region. The total number of carrier pairs produced including the primary electron that initiate the whole avalanche process is counted.

\section{B. Current Calculation}

This research applies the RPL model to estimate the time responses of avalanche photodiodes (APDs). It will consider an ideal structure which is assumed has a dimensionless multiplication length, $w=1.0$, with electrons and holes constant speeds, $v_{\mathrm{e}}=v_{\mathrm{h}}=v$, for various dead spaces distances, $d^{*}$. A computer code is generated to estimate the mean impulse response, $i(t)$, and standard deviation, $\sigma(t)$, of APDS.

In this simulation work, a double carrier multiplication APD with a multiplication region extending from $x=0$ until $x=w$ is under consideration. The avalanche process begins when an electron is introduced at position $x=0$. Due to the effect of electric field, it drifts in $x$ direction with a constant speed, $v_{\mathrm{e}}$. after the injection at a random distance, $l_{e}$, it initiates another impact ionization to generate an electron-hole pair. These successors carriers may start more ionization and produce more secondary carriers at random distances. The hole could perform the same process as the electron but in the reverse direction. The secondary electrons and holes repeat the process within the region. The impact ionization does not occur any more, when the electron arrives at the far-right side of the region (at position $x=w$ ) or the hole at the far-left side (at position $x=0$ ). When all the carriers left the region, as a result the avalanche process stopped. The 
random distance for a carrier to ionize is assumed to be statistically independent and have probability density function, $\mathrm{P}_{\mathrm{e}}(x)$ and $\mathrm{P}_{\mathrm{h}}(x)$, respectively, according to the random path length model explained in the previous section.

At location $x=0$ and time $t=0$, the avalanche process is started by injecting an electron. Then, the electric current is induced at time $t$, causing all carriers, electrons and holes, to move within the multiplication region. This current, which is a function of time, is a random impulse response function, $I(t)$, of the APD. The number of carriers generated are recorded in every time from the carrier injected time $(t=0)$ until the time when all carriers left the multiplication region. Afterward, the time response of current can be obtained from the following equation [12],

$I(t)=\frac{\mathrm{q}}{w}\left\{v_{e} N_{e}(t, 0)+v_{h} N_{h}(t, 0)\right\}$

where, $q$ is elementary charge, $w$ is multiplication region length, $v_{\mathrm{e}}$ and $v_{\mathrm{h}}$ are the electron and hole speed, respectively. $N_{\mathrm{e}}(t, 0)$ and $N_{\mathrm{h}}(t, 0)$ are the number of electron and hole at time $t$ produced by an injected electron at position $x=0$, respectively.

Since the electron speed is assumed to be constant and equal to the hole speed, $v_{\mathrm{e}}=v_{\mathrm{h}}=v$, then (8) becomes

$I(t)=\frac{\mathrm{q}}{w}\{v N(t, 0)\}$

where, $v$ is carrier speed and $N(t, 0)$ is the total number of carriers (electrons and holes) at time $t$ generated by injected electron at position $x=0$.

Many trials are performed to obtain more accurate data. In this estimation, 10000 trials are executed to get the mean impulse response,

$i(t)=\frac{1}{10000} \sum_{1}^{10000} I(t)$

After the mean impulse response, $i(t)$, has been determined, then its standard deviation can be calculated from the following relation

$\sigma(t)=\sqrt{\left\langle I^{2}(t)\right\rangle-i^{2}(t)}$

$\left\langle I^{2}(t)\right\rangle$ is the mean squared of time response of current. It can be obtained by raising power the random impulse response, $\mathrm{I}(t)$, to two and taking the average of the result.

\section{RESULTS AND DISCUSSIONS}

The mean impulse response, $i(t)$, and its standard deviation, $\sigma(t)$, are estimated for double carrier multiplication with electron and hole ionization coefficient, $\alpha$ and $\beta$ respectively, electron and hole dead space distance, $d_{e} *$ and $d_{h}{ }^{*}$ respectively, constant carrier speed, $v_{\mathrm{e}}=v_{\mathrm{h}}=v$. For a simplicity and better presentation, a normalized time $t^{*}=t /\left(w / v_{\mathrm{e}}\right)$ and mean scaled current $i^{*}\left(t^{*}\right)=\left(w / v_{\mathrm{e}}\right) \mathrm{i}\left(w t^{*} / v_{\mathrm{e}}\right)$ are defined.

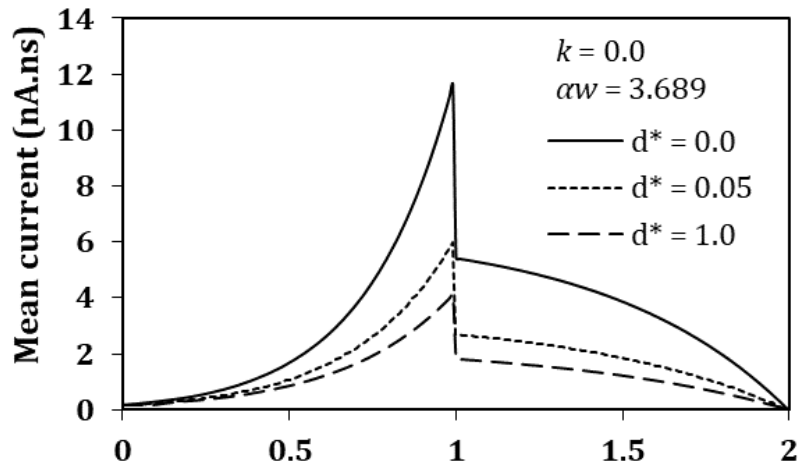

a) Normalized time
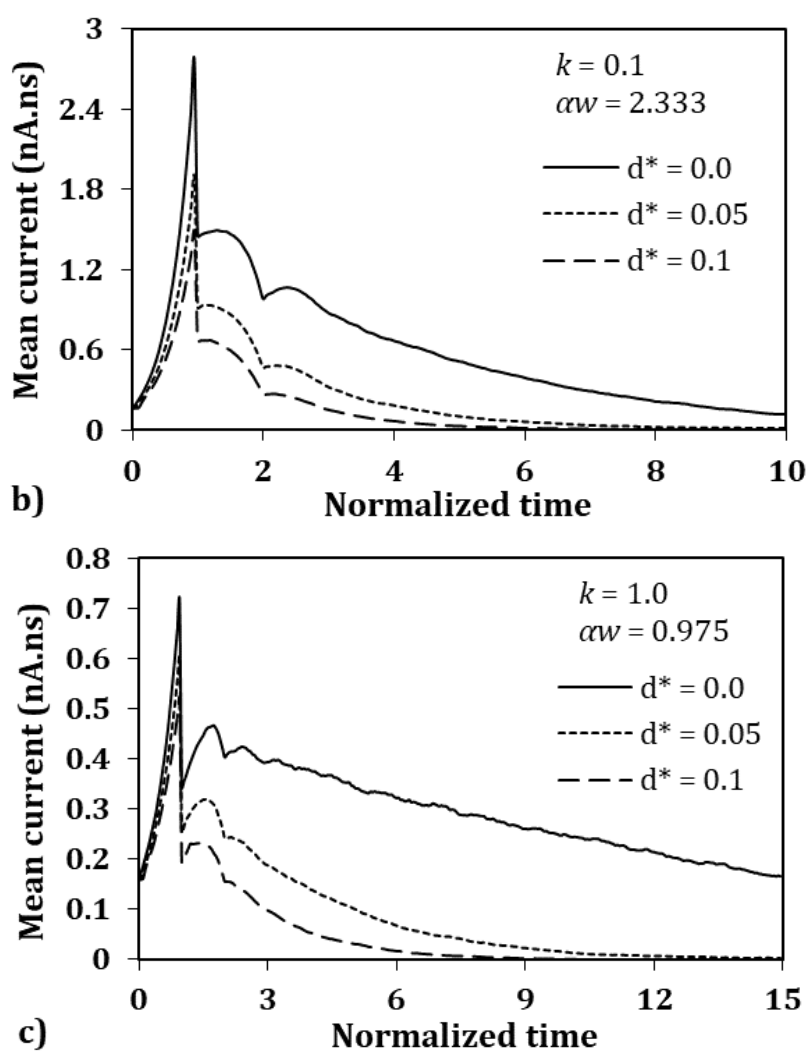

Figure 1. Scaled mean current, $i^{*}\left(t^{*}\right)=(w / v) \mathrm{i}\left(w \mathrm{t}^{*} / v\right)$, as a function of normalized time, $t^{*}=t /(w / v)$, for APDs with constant electron and hole speed, $v_{e}$ and $v_{h}$ respectively, for three cases of ionization ratio, a) $k=0.0$, b) $k=0.1$ and c) $k$ $=1.0$.

Mean scaled current, $i^{*}\left(t^{*}\right)$, for APDs with different values of hole-to-electron ionization coefficient ratios, $k=\beta / \alpha, 0.0,0.1$, and 1.0 are plotted in Fig. 1. All are evaluated for dead space ratio, $d_{e} * / w=d_{h} * / w=d^{*}=$ $0.0,0.05$, and 0.1 . The value of $\alpha w$ is selected to make multiplication gain, $<\mathrm{M}>$, at about 40 in the case of absence of dead space, $\alpha w=3.689(k=0.0), \alpha w=2.333$ $(k=0.1)$, and $\alpha w=0.975(k=1.0)$.

As can be seen from the Fig. 1, the increasing of dead space causes the reduction of mean impulse response, $i^{*}\left(t^{*}\right)$, for all time. Dead space causes the avalanche 
process to terminate faster. In the case of absence of dead space, the mean impulse response has wider profile compared to the others. Furthermore, the APDs with higher of hole-to-electron ratio, $k$, has longer of mean impulse response, this could be happened since both types of carrier give the same contribution into the avalanche process.
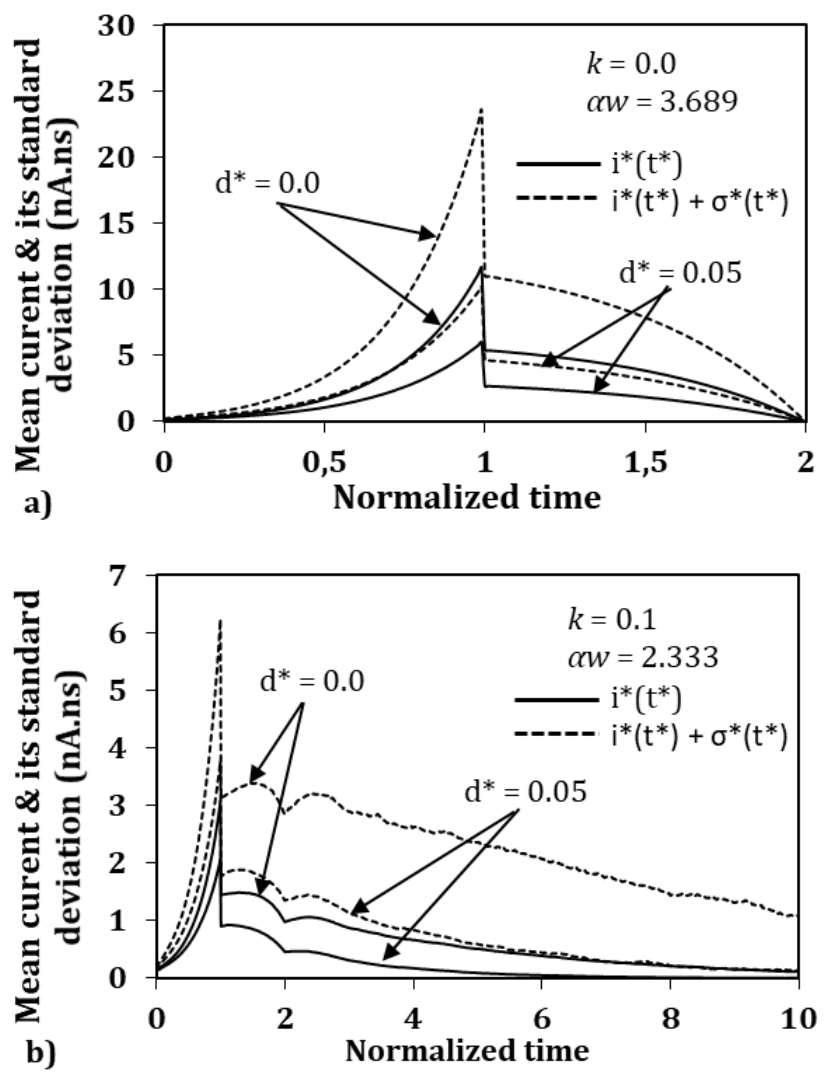

b) Normalized time

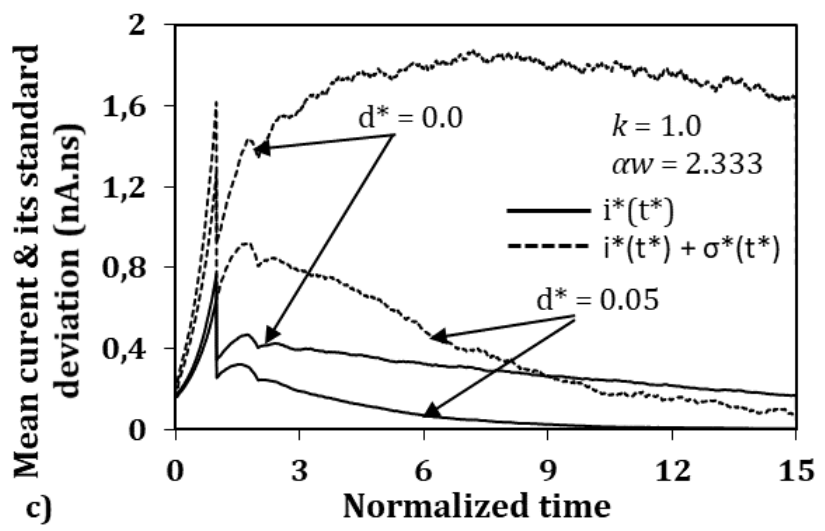

Figure 2. Scaled mean current, $i^{*}\left(t^{*}\right)$, together with scaled mean current plus its scaled standard deviation, $i^{*}\left(t^{*}\right)+$ $\sigma^{*}(t)$ as a function of normalized time, $t^{*}=t /(w / v)$, for

APDs with constant electron and hole speed, $v_{e}$ and $v_{h}$ respectively, in three cases of ionization ratio, a) $k=0.0$, b) $k=0.1$ and c) $k=1.0$.

Standard deviation of impulse response, $\sigma(t)$, is also computed to show the fluctuation of the impulse response. Fig. 2 shows the impulse response plus its standard deviation, $i^{*}\left(t^{*}\right)+\sigma^{*}\left(t^{*}\right)$, for hole-to-electron ionization ratios, $k=0.0,0.1$ and 1.0, and dead spaces, $d^{*}=0.0$ and 0.05 . The value of $\alpha \mathrm{w}$ is selected to obtain multiplication gain, $\langle\mathrm{M}\rangle$, at about 40 in the absence of dead space, $\alpha w=3.689(k=0.0), \alpha w=2.333(k=0.1)$, and $\alpha w=0.975(k=1.0)$.

It is shown that the scaled standard deviation, $\sigma\left(t^{*}\right)$, increases from the injection time $\left(t^{*}=0\right)$ until $t=\mathrm{w} / \mathrm{ve}$ or $t^{*}=1$, then decreases afterwards until the avalanche process terminated. In cases of same parameters of $k$ and $\alpha w$, dead space causes the reduction of $\sigma^{*}\left(t^{*}\right)$ for all time, $t^{*}$. As a result, the presence of dead space causes the reduction in the fluctuation of impulse response. These calculation results consent with previous results [7-8], which shows the effect of dead space on the decrease on the excess noise factor.

Furthermore, the RPL simulation results of time response parameters, i.e. mean impulse response and standard deviation of impulse response, of APDs are compared with those obtained by previous works [1114]. The comparison proves that RPL succeed to calculate the statistical properties of time response of APD as in this literature which develop the calculation based on solution of recurrence equations.

\section{CONCLUSION}

This paper presents a theoretical study of avalanche multiplication in Avalanche Photodiodes (APDs) by investigating the time response of APDs. The random path length model is developed to simulate the avalanche process and predict the mean impulse response, $i(t)$, and its standard deviation, $\sigma(t)$. The RPL models the carrier transport by developing random path lengths after impact ionization. It also takes account the dead space distance which is a minimum distance required by a carrier to gain sufficient energy to cause impact ionize. The estimation of time response is performed by assuming the carrier traveling in constant speed. The mean impulse response, $i(t)$, and its standard deviation, $\sigma(t)$, are plotted as a function of tome and calculated in three cases of ionization coefficient ratio, $k=0.0,0.1$ and 1.0, and three different dead space distances, $d^{*}=0.0,0.05 \mathrm{w}$ and $0.1 \mathrm{w}$.

Since electrons and holes contribute in the same portion in the avalanche process, the work result shows that APD with largest ionization ratio $(\mathrm{k}=1.0)$ has the longest mean impulse response. Since the dead space causes the avalanche process to terminate faster. it can be seen that the occurrence of dead space decreases the mean impulse response. In the case of no dead space, the mean impulse response has wider profile compared to others. Furthermore, the standard deviation estimation demonstrates that the presence of dead space has ability to reduce the fluctuation in the impulse current. 


\section{REFERENCES}

[1] G. P. Agrawal, Optical Receiver in Fiber-Optic Communications Systems, New York: John Wiley \& Sons, Inc, 2010.

[2] J. C. Campbell, "Recent Advances in Avalanche Photodiodes," J. Lightwave Technology, vol. 34, no. 2, pp. $278-285,2016$.

[3] A. Photodiode, "high sensitivity photodiodes having an internal gain mechanism," Hamamatsu Photonics K.K., Solid State Division, Hamamatsu, 2019.

[4] H. Zimmermann, "APD and SPAD Receivers: Invited Paper.," in Proceedings of 15th International Conference on Telecommunications, 2019.

[5] M. A. Saleh, M. M. Hayat, P. P. Sotirelis, A. L. Holmes, J. C. Campbell, B. E. A. Saleh and M. C. Teich, "Impact-Ionization and Noise Characteristics of Thin III-V Avalanche Photodiodes," IEEE Trans. Electron Devices, vol. 48, no. 12, pp. 2722-2731, 2001.

[6] A. R. J. Marshall, J. P. R. David and C. H. Tan, " Impact Ionization in InAs Electron Avalanche Photodiodes," IEEE Trans. Electron Devices, vol. 57, no. 10, pp. $2631-2638,2001$.

[7] J. S. Ng, C. H. Tan, B. K. Ng, P. J. Hambleton, J. P. R. David, G. J. Rees, A. H. You and D. S. Ong, "Effect of Dead Space on Avalanche Speed," IEEE Trans. Electron Devices, vol. 49, no. 4, pp. 544- 549, 2002.

[8] M. A. Saleh, M. M. Hayat, B. E. A. Saleh and M. C. Teich, "Dead-Space-Based Theory Correctly
Predicts Excess Noise Factor for Thin GaAs and AlGaAs Avalanche Photodiodes," IEEE Trans. Electron Devices, vol. 47, no. 3, pp. 625 -633, 2000.

[9] D. S. Ong, K. F. Li, G. J. Rees, J. P. R. David and P. N. Robson, "A Simple Model to Determine Multiplication and Noise in Avalanche Photodiodes," J. Applied Physics, vol. 83, no. 6, pp. 3426-3428, 1998.

[10] M. .. A. Spinelli and A. L. Lacaita, "Mean Gain of Avalanche Photodiodes in a Dead Space Model.," IEEE Trans. Electron Devices, vol. 43, no. 1, pp. 23-30, 1996.

[11] A. H. You and D. S. Ong, " Random Response Time of Thin Avalanche Photodiodes," Optical and Quantum Electronics, vol. 36, no. 13, pp. 1155 - $1166,2004$.

[12] P. J. Hambleton, S. A. Plimmer, J. P. R. David, G. J. Rees and G. M. Dunn, "Simulated Current Response in Avalanche Photodiodes," J. Applied Physics, vol. 91, no. 4, pp. 2107-2111, 2002.

[13] G. Kahraman, B. E. A. Saleh, W. L. Sargeant and M. C. Teich, "Time and Frequency Response of Avalanche Photodiodes with Arbitrary Structure," IEEE Trans. Electron Devices, vol. 39, no. 3, pp. 553 - 560, 1992.

[14] M. M. Hayat, O. H. Kwon, Y. Pan, P. Sotirelis, J. C. Campbell, B. E. A. Saleh and M. C. Teich., "Gain-Bandwidth Characteristics of Thin Avalanche Photodiodes," IEEE Trans. Electron Devices , vol. 49, no. 5, pp. 770-781, 2002. 